\title{
4 Metapsychologische Konzepte des Unbewussten
}

Das Unbewusste ist ein Kernbegriff der Psychoanalyse, dessen Genese man gut anhand der Modellvorstellungen des menschlichen Seelenlebens im Werk Freuds nachvollziehen kann. In einem ersten Kapitel werden die Konzeptionen des Unbewussten, wie Freud sie beschrieben hat, dargestellt. Im folgenden Abschnitt werden Arbeiten zum gesellschaftlich(en)/sozialen Unbewussten beleuchtet, soweit sie für die Zielsetzung dieser Arbeit von Bedeutung sind. Ein Verständnis der Funktionsweise des „,seelischen Apparates“ ist unabdingbar, um in weiterer Folge in einem dritten Abschnitt den Blick für die Äußerungsformen des Unbewussten, seine Repräsentationen, zu schärfen, die in Zusammenhang mit der ökonomischen Krise untersucht werden sollen.

\subsection{Freuds Modelle des Unbewussten}

Es gehört zu den Grundkonstanten des Freud'schen Denkens, dass ein Großteil unserer Seelentätigkeit unbewusst abläuft:

\begin{abstract}
„Die Unterscheidung des Psychischen in Bewußtes und Unbewußtes ist die Grundvoraussetzung der Psychoanalyse und gibt ihr allein die Möglichkeit, die ebenso häufigen als wichtigen pathologischen Vorgänge im Seelenleben zu verstehen, der Wissenschaft einzuordnen. Nochmals und anders gesagt: Die Psychoanalyse kann das Wesen des Psychischen nicht ins Bewußtsein verlegen, sondern muss das Bewußtsein als eine Qualität des Psychischen ansehen, die zu anderen Qualitäten hinzukommen oder wegbleiben darf" (S. Freud, 1923b, S. 283).
\end{abstract}

Damit unterschied Freud sich wesentlich von der „Bewusstseinspsychologie“ seiner Zeit, die für bestimmte psychische Phänomene, wenn man sie als Ausdruck des Bewusstseins zu verstehen versuchte, in einen Erklärungsnotstand geriet. Ja, fast in einer Umkehrung dazu schrieb Freud in der Traumdeutung:

„Das Unbewußte ist das eigentlich real Psychische, uns nach seiner inneren Natur so unbekannt wie das Reale der Außenwelt und uns durch die Daten des Bewußtseins ebenso unvollständig gegeben wie die Außenwelt durch die Angaben unserer Sinnesorgane" (S. Freud, 1900a, S. 580).

In Auseinandersetzung mit den wissenschaftlichen Strömungen seiner Zeit und in ständiger Umarbeitung seiner eigenen Erkenntnisse hat Freud seine Modellvorstellungen des Unbewussten entwickelt und differenziert.

(C) Der/die Autor(en) 2020

H. Klug, Die Finanzkrise 2008 im Unbewussten, https://doi.org/10.1007/978-3-658-28475-6_4 
In „Einige Bemerkungen über den Begriff des Unbewußten in der Psychoanalyse“ (S. Freud, 1912g) unterscheidet Freud die Begriffe „unbewusst“, „vorbewusst" und „bewusst".

Als bewusst gilt jene „Vorstellung, die in unserem Bewußtsein gegenwärtig ist und die wir wahrnehmen“ (S. Freud, 1912g, S. 29). „Eine unbewußte Vorstellung ist dann eine solche, die wir nicht bemerken, deren Existenz wir aber trotzdem auf Grund anderweitiger Anzeichen und Beweise zuzugeben bereit sind" (S. Freud, 1912g, S. 29).

Um den Unterschied zwischen vorbewussten und unbewussten Inhalten zu veranschaulichen, verweist Freud auf posthypnotische Aufträge. Menschen wird während der Hypnose eine Handlung aufgetragen, die diese nach dem Erwachen ausführen, ohne dass sie sich an den Auftrag erinnern können. Aber auch aufgrund seiner Analysen mit hysterischen Patienten kommt Freud zu der Überzeugung, dass es unbewusste Vorstellungen geben muss, „die nicht ins Bewußtsein dringen, wie stark sie auch sein mögen" (S. Freud, 1912g, S. 31). Während vorbewusste Inhalte einen Widerstand überwinden und ins Bewusstsein gelangen können, gelingt dies unbewussten Inhalten nicht.

Damit kommt der dynamische, das heißt der wirksame Charakter dieser Vorstellungen ins Spiel.

„So lernen wir, dass der unbewußte Gedanke vom Bewußtsein durch lebendige Kräfte ausgeschlossen wird, die sich seiner Annahme entgegenstellen, während sie anderen Gedanken, den vorbewußten, nichts in den Weg legen " (S. Freud, 1912g, S. 33).

Freud weist in diesem Zitat darauf hin, dass es sich hierbei nicht bloß um gewisse Funktionsstörungen bei Kranken handelt, sondern dass jeder von uns von solchen Vorgängen Zeugnis ablegen kann.

„[...] Lapsus linguae, Gedächtnis- und Sprachirrtümer, Namenvergessen usw. können leicht auf die Wirksamkeit starker unbewußter Gedanken zurückgeführt werden, geradeso wie die neurotischen Symptome" (S. Freud, 1912g, S. 33).

Anhand der Traumbildung zeigt er, wie sich Tagesreste mit den wirksamen unbewussten Wünschen aus der Kindheit verbinden und eine Umbildung erfahren können, die schließlich bewusst wird (vgl. S. Freud, 1912g, S. 34f.).

Dieser Bereich des Unbewussten unterliegt eigenen Gesetzmäßigkeiten, auf die noch näher eingegangen werden wird. Was Freud in diesem Aufsatz leistet, ist, den Begriff ,unbewusst" in seinen deskriptiven, dynamischen - das heißt wirksamen - und systematischen Aspekten zu beschreiben. Wird der Begriff systematisch gebraucht, werden die Abkürzungen $B w$ für Bewusstsein und $U b w$ für Unbewusstes von Freud vorgeschlagen (vgl. S. Freud, 1915e, S. 131). 
In „Das Unbewußte“ (S. Freud, 1915e) entwickelt Freud eine Topographie der seelischen Vorgänge, eine „Tiefenpsychologie“ (S. Freud, 1915e, S. 132), die nicht anatomisch aufgefasst werden darf, sondern als Modell von den Funktionsweisen der Psyche verstanden werden muss. „Unsere psychische Topik hat vorläufig nichts mit der Anatomie zu tun; sie bezieht sich auf Regionen des seelischen Apparats, wo immer sie am Körper gelegen sein mögen, und nicht auf anatomische Örtlichkeiten“" (S. Freud, 1915e, S. 133).

Freud grenzt hier die Bereiche des Seelenlebens ab, charakterisiert ihre Eigentümlichkeiten, den Verkehr zwischen den einzelnen Bereichen sowie die spezifischen Wirkmechanismen und stellt diese in einen Zusammenhang mit den Ergebnissen der Behandlung seiner Patienten, da die Psychoanalyse es ermöglicht, Einfluss auf das Unbewusste zu nehmen, wenn dies auch ein schwieriges und lange dauerndes Unternehmen ist (vgl. S. Freud, 1915e, S. 153).

„Den Inhalt des Ubw kann man mit einer psychischen Urbevölkerung vergleichen. Wenn es beim Menschen ererbte psychische Bildungen, etwas dem Tiere Analoges gibt, so macht dies den Kern des Ubw aus "(S. Freud, 1915e, S. 154).

Dieser Kern „,besteht aus Triebrepräsentanzen, die ihre Besetzung abführen wollen, also aus Wunschregungen“" (S. Freud, 1915e, S. 145). Das heißt, der Trieb selbst kann bloß über eine Vorstellungsrepräsentanz oder über einen Affekt ins Bewusstsein gelangen (vgl. S. Freud, 1915e, S. 136). Im Unbewussten gibt es „keine Negation, keinen Zweifel, keine Grade von Sicherheit“ (S. Freud, 1915e, S. 145). „Die Vorgänge des Systems Ubw sind zeitlos [...]“ (S. Freud, 1915e, S. 145).

Der Begriff der Besetzung ist in diesem Zusammenhang ein bedeutsamer und verweist auf den mechanisch-energetischen Hintergrund der Modellvorstellung, in der es um die Verteilung von Energie geht, genauer gesagt, um die Energie der Sexualtriebe. ${ }^{42}$ Damit erschließt sich für Freud die Möglichkeit, neben der dynamischen und topischen auch eine ökonomische Betrachtungsweise der psychischen Phänomene einzuführen, ,welche die Vollendung der psychoanalytischen Forschung ist“ (S. Freud, 1915e, S. 140). Er schreibt: „Ich schlage vor, daß es eine metapsychologische Darstellung genannt werden soll" (S. Freud, 1915 e, S. 140).

Wie sehr das Freud'sche Denken von ökonomischen Vorstellungen geprägt ist, wäre wert, es in einer eigenen Arbeit zu untersuchen.

Während das Unbewusste primärprozesshaften Charakter hat (Beweglichkeit der Besetzungen), unterliegt das Bewusste dem Sekundärvorgang (zeitliche

42 „Wir dürfen dabei ,Besetzung“ durch ,Libido“ ersetzen, weil es sich ja, wie wir wissen, um die Schicksale von Sexualtrieben handelt" (S. Freud, 1915e, S. 141). 
Anordnung der Vorstellungsinhalte, Zensur, Realitätsprüfung oder Realitätsprinzip) (vgl. S. Freud, 1915e, S. 147). Wie sehr es dem Bewusstsein gelingt, die Affektentbindung zu hemmen und die Herrschaft über die Motilität zu sichern, entscheidet darüber, ob sich das Individuum im Zustand der Neurose, Psychose oder Normalität befindet (vgl. S. Freud, 1915e, S. 138).

Der Verteilung dieser Energie, ihr Abzug, ihre Verschiebung oder Verdichtung sind bestimmend für das „Schicksal“ von Vorstellungen, darüber, ob sie dem Bereich des Unbewussten, Vorbewussten oder Bewussten angehören. Man darf sich nach Freud diese Bereiche nicht wie voneinander abgeschlossene Systeme vorstellen, sondern eher wie kommunizierende Gefäße.

„Das Ubw ist vielmehr lebend, entwicklungsfähig und unterhält eine Anzahl von anderen Beziehungen zum Vbw, darunter auch Kooperation" (S. Freud, 1915e, S. 149). „Der Inhalt des Systems Vbw (oder Bw) entstammt zu einem Teile dem Triebleben [...], zum anderen Teile der Wahrnehmung " (S. Freud, 1915e, S. 153).

Zwischen den einzelnen Systemen besteht eine Art von Grenze - Freud nennt sie Zensur -, die darüber entscheidet, welche Vorstellungen oder Affekte über das Vorbewusste in das Bewusste übergehen dürfen.

„,Wir tun aber gut daran [...] anzunehmen, dass jedem Übergang von einem System zum nächst höheren, also jedem Fortschritt zu einer höheren Stufe der Organisation eine neue Zensur entspreche“ (S. Freud, 1915e, S. 150). „Alle Wege von der Wahrnehmung zum Ubw bleiben in der Norm frei; erst die vom Ubw weiterführenden Wege unterliegen der Sperrung durch die Verdrängung “ (S. Freud, 1915e, S. 153).

Der Begriff der Verdrängung ist sozusagen der dynamische Aspekt der Zensur und wird in seiner Wirkungsweise folgendermaßen beschrieben:

Die Verdrängung, die ,sich an Vorstellungen an der Grenze der Systeme Ubw und Vbw (Bw) vollzieht" (S. Freud, 1915e, S. 139), entzieht diesen ihre Besetzung. ${ }^{43}$ Diese Vorstellungen bleiben aber, wie Freud schreibt, im Unbewussten aktionsfähig. Was ihnen entzogen wurde, muss daher die vorbewusste Besetzung sein (vgl. S. Freud, 1915e, S. 139). „Die Vorstellung bleibt dann unbesetzt, oder sie erhält Besetzung vom Ubw her, oder sie erhält die ubw Besetzung, die sie schon früher hatte“" (S. Freud, 1915e, S. 139).

Weiter unten präzisiert Freud, was das genaue Unterscheidungsmerkmal zwischen den Vorstellungen in beiden Systemen ist:

43 Unter Besetzung versteht man, ,daß eine bestimmte psychische Energie an eine Vorstellung oder Vorstellungsgruppe, einen Teil des Körpers, ein Objekt etc. gebunden ist" (Laplanche \& Pontalis, 1989, S. 92). 
„Die beiden sind nicht, wie wir gemeint haben, verschiedene Niederschriften desselben Inhalts an verschiedenen psychischen Orten, auch nicht verschiedene funktionelle Besetzungszustände an demselben Orte, sondern die bewußte Vorstellung umfaßt die Sachvorstellung plus der dazugehörigen Wortvorstellung, die unbewußte ist die Sachvorstellung allein" (S. Freud, 1915e, S. 160).

Die Beschreibung dieses Vorganges wirft unweigerlich die Frage nach der Genese der beiden Bereiche, der Teilung in Bewusstes und Unbewusstes, auf. Freud löst sie, indem er einen Vorgang konstatiert, den er Urverdrängung nennt, die durch eine „Gegenbesetzung“ zustande gekommen ist und „durch welche sich das System Vbw gegen das Andrängen der unbewussten Vorstellung schützt“" (S. Freud, 1915e, S. 140).

Was die Affekte betrifft, können sie ein dreifaches Schicksal erleiden:

„[...] der Affekt bleibt entweder - ganz oder teilweise - als solcher bestehen, oder er erfährt eine Verwandlung in einen qualitativ anderen Affektbetrag, vor allem in Angst, oder er wird unterdrückt, $d$. h. seine Entwicklung überhaupt verhindert" (S. Freud, 1915e, S. 137).

Nach Freud ist ,[...] die Unterdrückung der Affektentwicklung das eigentliche Ziel der Verdrängung“ (S. Freud, 1915e, S. 137).

Sogenannten „Abkömmlingen des Unbewussten“, die Merkmale beider Systeme - allerdings verschleiert - tragen, gelingt es immer wieder, die Zensur zu passieren, wenn sie zum Beispiel mit einer Gegenbesetzung des Vorbewussten zusammentreffen. Freud vergleicht sie mit den ,Mischlingen menschlicher Rassen [...], die im Großen und Ganzen bereits den Weißen gleichen, ihre farbige Abkunft aber durch den einen oder anderen auffälligen Zug verraten und darum von der Gesellschaft ausgeschlossen bleiben und keines der Vorrechte der Weißen genießen“ (S. Freud, 1915e, S. 149 f.). Dazu zählen zum Beispiel bestimmte Symptome, wie sie bei Phobien auftreten, oder die manifesten Traumgedanken.

Wenn man das Zusammenwirken der Systeme, ihren Austausch und ihre Genese betrachtet, wird der Begriff Bewusstheit zusehends problematisch.

„Abgesehen davon, daß das Bewußte nicht immer bewußt, sondern zeitweilig auch latent ist, hat uns die Beobachtung gezeigt, daß vieles, was die Eigenschaften des Systems Vbw teilt, nicht bewußt wird, und haben wir noch zu erfahren, daß das Bewußtwerden durch gewisse Richtungen seiner Aufmerksamkeit eingeschränkt ist " (S. Freud, 1915e, S. 151 ).

Bereits 1915 verweisen dieser Zweifel und der Gedanke, dass ,nicht nur das psychisch Verdrängte dem Bewußtsein fremd bleibt, sondern auch ein Teil der unser Ich beherrschenden Regungen, also der stärkste funktionelle Gegensatz des 
Verdrängten“"(S. Freud, 1915e, S. 151), auf die Notwendigkeit einer Neukonzeption des Modells:

„Es würde allen Mißverständnissen ein Ende machen, wenn wir von nun an bei der Beschreibung der verschiedenartigen psychischen Akte ganz davon absehen würden, ob sie bewußt oder unbewußt sind, und sie bloß nach ihrer Beziehung zu den Trieben und Zielen, nach ihrer Zusammensetzung und Angehörigkeit zu den einander übergeordneten psychischen Systemen klassifizieren und in Zusammenhang bringen würden" (S. Freud, 1915e, S. 131).

Im Jahr 1923 legt Freud mit dem Aufsatz „Das Ich und das Es“ (1923b) ein „Strukturmodell“ der menschlichen Psyche vor, mit dem er jene Schwierigkeiten $\mathrm{zu}$ beseitigen versucht, die bei der topischen Betrachtungsweise noch keiner Lösung zugeführt werden konnten. In diesem Aufsatz wird zwischen Ich, Es und Über-Ich als unterschiedlichen psychischen Strukturen unterschieden.

Das Ich ist eng an die Funktion der Wahrnehmung geknüpft ${ }^{44}$, allerdings erschöpft es sich nicht darin. Freud schreibt, auf Groddeck bezogen, der ein Buch mit dem Titel „Das Buch vom Es“" verfasst hat:

„Ich schlage vor, [...] das vom System $W$ ausgehende Wesen, das zunächst vbw ist, das Ich zu heißen, das andere Psychische aber, in welches es sich fortsetzt und das sich wie ubw verhält, nach Groddecks Gebrauch das Es “ (S. Freud, 1923b, S. 292).

In Abbildung 5 erkennt man das Ich als Teil des Es, unter dem Einfluss der Wahrnehmung stehend, durch eine Schranke von den verdrängten Inhalten getrennt, die nur einen Teil des Unbewussten ausmachen. ${ }^{45}$

44 Freud macht deutlich, dass der Unterschied zwischen einer vorbewussten Vorstellung und einer unbewussten darin begründet ist, dass erstere eine Verbindung mit bestimmten Wortvorstellungen eingehen kann. „Diese Wortvorstellungen sind Erinnerungsreste, sie waren einmal Wahrnehmungen [...]: bewußt werden kann nur das, was schon einmal bw Wahrnehmung war, und was außer Gefühlen von innen her bewußt werden will, muß versuchen sich in äußere Wahrnehmung umzusetzen" (S. Freud, 1923b, S. 289). Dabei handelt es sich vorwiegend um akustische und erst zweitrangig um visuelle Erinnerungen (vgl. S. Freud, 1923b, S. 289 f.).

45 Freud verweist darauf, „dass es im deskriptiven Sinn zweierlei Unbewußtes gibt, im dynamischen Sinn nur eines“" (S. Freud, 1923b, S. 285). Damit ist gemeint, dass das dynamisch Unbewusste sich auf das Verdrängte bezieht, das deskriptiv Unbewusste bewusstseinsfähige und nicht bewusstseinsfähige Inhalte umfasst. „Wir erkennen, daß das Ubw nicht mit dem Verdrängten zusammenfällt; es bleibt richtig, daß alles Verdrängte ubw ist, aber nicht alles Ubw ist auch verdrängt" (S. Freud, 1923b, S. 287). 


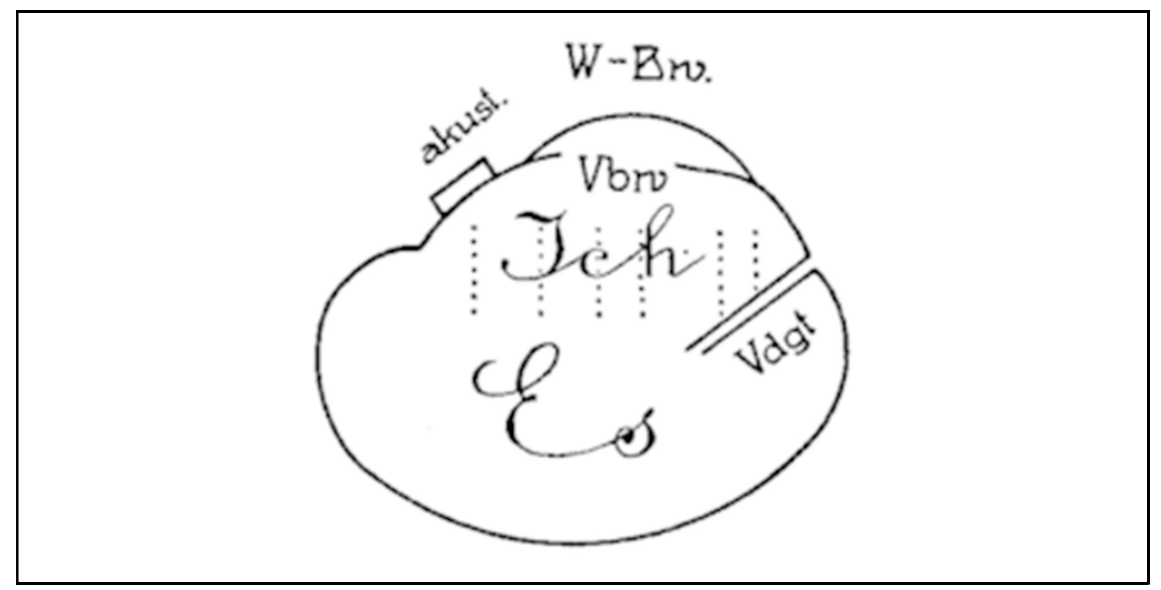

Abbildung 5: $\quad$ Das Ich und das Es (S. Freud, 1923b, S. 293)

„Das Ich ist vor allem ein körperliches, es ist nicht nur ein Oberflächenwesen, es ist selbst die Projektion einer Oberfläche“ (S. Freud, 1923b, S. 294), schreibt Freud. Die Körperoberfläche ist mit ihren Sinnesorganen eine Quelle von inneren Wahrnehmungen, das heißt Empfindungen, und äußeren Wahrnehmungen, die auf das Ich einströmen. Was seine Funktionen betrifft,

„[...] stellt es die zeitliche Anordnung der seelischen Vorgänge her und unterzieht dieselben der Realitätsprüfung. Durch die Einschaltung der Denkvorgänge erzielt es einen Aufschub der motorischen Entladungen und beherrscht die Zugänge zur Motilität" (S. Freud, 1923b, S. 321).

Greift man das Bild vom Ich als Oberflächenwesen auf, erkennt man auch bald die Schwierigkeiten, die mit dieser Situierung verbunden sind:

„In seiner Mittelstellung zwischen Es und Realität unterliegt es nur zu oft der Versuchung, liebedienerisch, opportunistisch und lügnerisch zu werden, etwa wie ein Staatsmann, der bei guter Einsicht sich doch in der Gunst der öffentlichen Meinung behaupten will“ (S. Freud, 1923b, S. 323).

Dabei ist die prekäre Situation des Ich noch nicht vollständig charakterisiert. Gerade weil das Ich unter dem Einfluss der Außenwelt aus dem Es entstanden ist und seine Herkunft nicht verleugnen kann, wenn Abkömmlinge der Triebe trotz Zensur in seinen Bereich vordringen, wird es auch von dieser Seite massiv unter Druck gesetzt: „Wie dem Reiter, will er sich nicht vom Pferd trennen, oft nichts anderes übrigbleibt, als es dahin zu führen, wohin es gehen will, so pflegt auch 
das Ich den Willen des Es in Handlung umzusetzen, als ob es der eigene wäre" (S. Freud, 1923b, S. 294).

Was in Abbildung 5 nicht zu sehen ist, ist eine weitere Differenzierung im Ich, die Freud das Über-Ich nennt. Damit wird das Ich endgültig zu einem Diener dreier Herren, „welches unter dreierlei Dienstbarkeiten steht und demzufolge unter den Drohungen von dreierlei Gefahren leidet, von der Außenwelt her, von der Libido des Es und von der Strenge des Über-Ichs“" (S. Freud, 1923b, S. 322).

„,Während das Ich wesentlich Repräsentant der Außenwelt, der Realität ist, tritt ihm das Über-Ich als Anwalt der Innenwelt, des Es gegenüber. Konflikte zwischen Ich und Ideal werden [...] in letzter Linie den Gegensatz von Real und Psychisch, Außenwelt und Innenwelt widerspiegeln " (S. Freud, 1923b, S. 303).

Das Über-Ich, das Freud in diesem Aufsatz mit dem Ichideal gleichsetzt, wird durch Identifizierungen gebildet. So ist das Über-Ich einerseits ein Erbe der Identifizierung mit dem „Vater der persönlichen Vorzeit““46 (S. Freud, 1923b, S. 298 f.): Freud geht von einem phylogenetischen Erbe aus, das im Es verankert ist. Wie er in „Totem und Tabu“ (S. Freud, 1912-13a ) darstellt, wird durch den Mord am Urvater, der die Macht über die Horde, die Frauen und Nachkommen hat, ein neues Gesetz etabliert. Mit dem Inzesttabu und der Installierung eines Totems werden die Beziehungen zwischen den Söhnen so geregelt, dass sich nicht einer von ihnen selbst zum neuen Übervater aufschwingen kann. Dem Totem kommt mehr Macht zu als dem Urvater. Das, was sich im Über-Ich widerspiegelt, ist sowohl eine durch Ambivalenz bedingte Unterwerfung als auch, wie weiter unten dargestellt werden wird, die Rebellion gegen dieses Ideal. $^{47}$

Andererseits ist das Über-Ich ein Erbe des Ödipuskomplexes ${ }^{48}$, das heißt, ein Ergebnis der jeweils individuellen Entwicklung eines jeden Menschen, in dem sich ein überindividuelles Muster wiederholt. Was passiert, ist, dass das

46 In einer Fußnote zu dieser Formulierung schreibt Freud: „Vielleicht wäre es vorsichtiger zu sagen, mit den Eltern, denn Vater und Mutter werden vor der sicheren Erkenntnis des Geschlechtsunterschiedes, des Penismangels, nicht verschieden gewertet

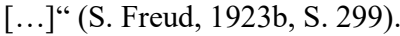

47 Die Weitergabe dieser Grunderfahrungen über Generationen ist eine unbewusste und gehört dem Es an: „Somit beherbergt das erbliche Es in sich die Reste ungezählt vieler Ich-Existenzen, und wenn das Ich sein Über-Ich aus dem Es schöpft, bringt es vielleicht nur ältere Ichgestaltungen wieder zum Vorschein, schafft ihnen eine Auferstehung“" (S. Freud, 1923b, S. 305).

48 Freud bezieht sich in seinen Ausführungen auf die Ödipussage. In der positiven Variante zeigt sich der Ödipuskomplex durch die Todeswünsche des Kindes gegenüber dem gleichgeschlechtlichen Elternteil und die sexuellen Wünsche gegenüber dem gegengeschlechtlichen Elternteil. In der negativen Variante ist dies umgekehrt. 
Über-Ich als Instanz gegen die frühen Objektwahlen des Kindes aufgerichtet wird, gegen die liebevollen Strebungen des Knaben in Bezug auf die Mutter, des Mädchens in Bezug auf den Vater (in der einfachen Variante ${ }^{49}$ des Ödipuskomplexes), und die abgezogene Besetzungsenergie von den Objektwahlen auf das Ichideal gerichtet wird, dem das Ich nun zu gefallen trachtet. Dabei wird der ambivalente Charakter des Über-Ichs gegenüber dem Ich offenkundig: „So (wie der Vater) sollst du sein [...] So (wie der Vater) darfst du nicht sein, das heißt nicht alles tun, was er tut; manches bleibt ihm vorbehalten“ (S. Freud, 1923b, S. 301 f.).

Spätere Identifizierungen mit Autoritäten stärken dieses Ideal, das, wie Freud schreibt, „den Keim, aus dem sich alle Religionen gebildet haben“ (S. Freud, 1923b, S. 304) enthält und neben Religion und Moral auch die Basis für das soziale Empfinden bildet, das „als Überbau über die eifersüchtigen Rivalitätsregungen gegen die Geschwister" (S. Freud, 1923b, S. 304) angesehen werden kann. Es äußert sich in schlechtem Gewissen und in Schuldgefühlen (vgl. S. Freud, 1923b, S. 304). Die Stärke dieser Gefühle und die Unerbittlichkeit der Kritik basieren auf den Verschiebungen von Energie auf der Triebebene, die letztlich das Ich, das zwischen den Instanzen zu vermitteln sucht, in arge Bedrängnis bringen können.

Freud unterscheidet zu diesem Zeitpunkt zwischen zwei Triebformen, zwischen dem Sexualtrieb oder Eros und dem Todestrieb, die beide unterschiedliche Ziele verfolgen: Während der Sexualtrieb nach Synthese strebt und den Fortgang des Lebens zum Ziel hat, verfolgt der Todestrieb das Ziel, „das organische Leben in den leblosen Zustand zurückzuführen“" (S. Freud, 1923b, S. 307). Diese beiden im theoretischen Modell getrennten Komponenten muss man real jedoch als stets gemischt denken („Triebmischung“).

Wenn man nun die Beziehung der drei Strukturen Es, Ich und Über-Ich zu den Trieben genauer betrachtet, kann man sagen, dass das Es der Ort der Triebe ist, „Eros und Todestrieb kämpfen in ihm“ (S. Freud, 1923b, S. 325).

Das Ich selbst muss sich gegen die Abkömmlinge der Triebe wehren. Freud beschreibt die Beziehung des Ich zum Es folgendermaßen:

„Zu Uranfang ist alle Libido im Es angehäuft, während das Ich noch in der Bildung begriffen und schwächlich ist. Das Es sendet einen Teil dieser Libido auf erotische Objektbesetzungen aus, worauf das erstarkte Ich sich dieser Objektlibido zu bemächtigen und sich dem Es als Liebesobjekt aufzudrängen versucht. Der Narzissmus des Ichs ist so ein sekundärer, den Objekten entzogener" (S. Freud, 1923b, S. 313).

49 Freud merkt kritisch an, dass diese einfache Variante, eine schematische Darstellung, praktischen Gründen geschuldet ist. Wegen der Bisexualität des Kindes besteht die vollständige Variante aus einem positiven und einem negativen Ödipuskomplex (vgl. S. Freud, 1923b, S. 300 f.). 
Auf der anderen Seite wird das Ich von den Anforderungen des Über-Ichs stark bedrängt. Die Mischung von Eros und Todestrieb spielt eine entscheidende Rolle dabei, wie es agiert. Das Über-Ich selbst ist, wie weiter oben dargestellt, tief im Es verwurzelt, weil es dem Ödipuskomplex einen Gutteil seiner Entstehung verdankt.

„Das Über-Ich ist ja durch eine Identifizierung mit dem Vatervorbild entstanden. Jede solche Identifizierung hat den Charakter einer Desexualisierung oder selbst Sublimierung. Es scheint nun, daß bei einer solchen Umsetzung auch eine Triebentmischung stattfindet [...] Die erotische Komponente hat nach der Sublimierung nicht mehr die Kraft, die ganze hinzugesetzte Destruktion zu binden, und diese wird als Aggressions- und Destruktionsneigung frei. Aus dieser Entmischung würde das Ideal überhaupt den harten, grausamen Zug des gebieterischen Sollens beziehen" (S. Freud, 1923, S. 321).

So wird gerade durch die Triebentmischung die Unerbittlichkeit und Härte des Über-Ichs gegenüber dem Ich verständlich.

Nun wird deutlich, welch große Anpassungsleistung vonseiten des Ichs notwendig ist, um den Geboten des Über-Ichs und damit den tradierten gesellschaftlichen Regeln und Anforderungen der Kultur Genüge zu tun. Die Wendung der Aggression nach innen oder nach außen ist eng mit der Sublimierungsfähigkeit des Individuums verknüpft; je sublimierungsfähiger das Individuum ist, desto mehr kehrt es die Aggression gegen sich: „Der gewöhnlichen Betrachtung erscheint dies umgekehrt, sie sieht in der Forderung des Ichideals das Motiv für die Unterdrückung der Aggression“ (S. Freud, 1923b, S. 320 f.). „Es ist wie eine Verschiebung, eine Wendung gegen das eigene Ich" (S. Freud, 1923b, S. 321).

Aus den unterschiedlichen Kräfteverhältnissen dieser Strukturen, die letztlich alle aus dem Es entstanden sind, lassen sich Erkenntnisse über die neurotischen Erkrankungen gewinnen, wobei sich das Ich in der Hysterie gegenüber dem Es und dem Über-Ich vorwiegend mittels Verdrängung, bei der Zwangsneurose hauptsächlich mittels Reaktionsbildungen zur Wehr setzt. In der Melancholie jedoch ist es dem Über-Ich schutzlos ausgeliefert.

„Durch seine Identifizierungs- und Sublimierungsarbeit leistet es den Todestrieben im Es Beistand zur Bewältigung der Libido, gerät aber dabei in Gefahr, zum Objekt der Todestriebe zu werden und selbst umzukommen. Es hat sich zu Zwecken der Hilfeleistung selbst mit Libido erfüllen müssen, wird dadurch selbst Vertreter des Eros und will nun leben und geliebt werden " (S. Freud, 1923b, S. 323).

Der gravierende Unterschied zwischen den verschiedenen Endgestaltungen der Erkrankungen liegt in den Objektbesetzungen, die bei Hysterie und Zwangsneurose bestehen bleiben, bei der Melancholie jedoch abgezogen und durch Identifizierung ins Ich eingeschrieben werden (vgl. S. Freud, 1923b, S. 317 f.). 
Am Ende des Textes stellt Freud die Angst in den Fokus seiner Betrachtungen und macht das Ich zur eigentlichen Stätte der Angst (vgl. S. Freud, 1923b, S. 323), weil es sich gegenüber den äußeren Gefahren, dem Triebgeschehen und dem Über-Ich schützen muss. Die Angst vor Letzterem ist eigentlich eine Kastrationsangst, „um die sich die spätere Gewissensangst ablagert" (S. Freud, 1923b, S. 324), womit wieder eine Verbindung zum Ödipuskomplex hergestellt wird. Zur Todesangst wird sie dann, wenn es sich um eine äußere Gefahr oder um die Gefahr des Verlustes der Liebe des Über-Ichs wie bei der Melancholie handelt. Den Schutz der Elternfiguren, ihre Liebe zu verlieren, bedeutet den Tod des Ichs:

„Es sieht sich von allen schützenden Mächten verlassen und lässt sich sterben. Es ist übrigens immer noch dieselbe Situation, die dem ersten großen Angstzustand der Geburt und der infantilen Sehnsucht-Angst zugrunde lag, die der Trennung von der schützenden Mutter" (S. Freud, 1923b, S. 324 f.).

Aus diesen Darlegungen ist ersichtlich, dass die Unterteilung in Bewusstes und Unbewusstes nicht mit den einzelnen Strukturen konform geht, dass verdrängte Inhalte bewusst gemacht werden können, wenn sie die Verdrängungsschranke passieren, dass einzelne Tätigkeiten des Ichs wie zum Beispiel die Abwehrtätigkeit unbewusst erfolgen und das Über-Ich aufgrund seiner tiefen Beziehung zum Es viele unbewusste Anteile aufweist.

„Nicht nur das Tiefste, auch das Höchste am Ich kann unbewußt sein. Es ist, als würde uns auf diese Weise demonstriert, was wir vorhin vom bewußten Ich ausgesagt haben, es sei vor allem ein Körper-Ich" (S. Freud, 1923b, S. 295).

In der Zeit nach Freud wurden die Konzeptionen und Vorstellungen des Unbewussten weiterentwickelt.

„Im kleinianischen Modell finden wir neben dieser vertikalen Metaphorik einen stärker horizontal aufgespannten metaphorischen Raum, in dem sich die unbewussten Prozesse der projektiven Identifizierung entfalten. Teile des Selbst wandern in den Anderen als Objekt hinein, so wie auch Teile des Anderen ins Selbst assimiliert werden. Anders, aber metaphorisch doch ähnlich verhält es sich mit der Dialektik von Selbst und Anderem etwa bei Jean Laplanche. Bei ihm wird das Unbewusste des Kindes zum Ort der rätselhaften, sexuell unterfütterten Botschaften des Anderen, d.h. vor allem der Mutter. In einem dritten Modell, das den neueren intersubjektiven Theorien entstammt, ist die interpersonale Beziehung selbst Ort des Unbewussten " (Bohleber, 2013, S. 807).

Der vorliegenden Arbeit wird das Strukturmodell Freuds zugrundegelegt, es wird aber auch auf Weiterentwicklungen Bezug genommen, sofern diese dem Verständnis der untersuchten psychischen Prozesse dienen. So hat die kleinianische 
Schule besonders die Rolle der unbewussten Phantasien hervorgehoben und manische und depressive Positionen als Stadien der Persönlichkeitsentwicklung und Organisation von Gedanken und Emotionen bei jedem Erwachsenen postuliert.

Freud beschreibt in seinem Strukturmodell die enge Verbindung von individuellen und gesellschaftlichen Aspekten, die durch das Erbe der Vorzeit, das von Generation zu Generation weitergegeben wird, im Über-Ich ihren Niederschlag finden und die sozialen Beziehungen bestimmen und regeln. An Freuds Modell der Genese des Über-Ichs, dessen transgenerationalen Verankerung in Kultur und Gesellschaft, wird deutlich, dass die Kritik, die Psychoanalyse vernachlässige soziale Aspekte oder habe nur einen begrenzten Blickwinkel darauf, ins Leere geht. Bernfeld schreibt:

„Der Einwand selbst ist alt; er hatte sich früher in die Formel gekleidet, ,solche Dinge, wie Freud sie behaupte, gäbe es nur im sittenlosen Wien ' [...] Sehen wir von der gelegentlich kindischen polemischen Wendung dieses Vorwurfs ab, so trifft er doch einen Wahrheitskern. Die Psychoanalyse hat sich noch nicht gründlich genug mit den sozialen Beziehungen all jener seelischen Vorgänge befasst, die sie entdeckte und ausführlich studierte" (Bernfeld, 1974a, S. 209).

Ich möchte daher die Konzeptionen des individuellen Unbewussten durch die Modelle des gesellschaftlich/-en Unbewussten und sozialen Unbewussten ergänzen, die in den letzten Jahren und Jahrzehnten entwickelt wurden. Die Erfahrungen aus der Arbeit mit Gruppen trugen entscheidend dazu bei, eine differenzierte Sicht auf Prozesse zu richten, die den wechselseitigen Einfluss von Individuen, Gruppen und größeren Gemeinschaften beschreiben.

\subsection{Modelle des gesellschaftlich/-en ${ }^{50}$ und sozialen Unbewussten}

"The first difficulty that the notion of the social unconscious seeks to compensate for, is the absence of the social in much psychoanalytic discourse" (Dalal, 2001).

In diesem Kapitel werden unterschiedliche Konzepte des gesellschaftlich/-en und sozialen Unbewussten beschrieben. Die Wurzeln dieser Modelle reichen in die Blütezeit der Psychoanalyse zurück; hier sind die Arbeiten Fromms und Bernfelds zu nennen. Die Konzepte wurden nach dem Krieg besonders im Bereich

50 Erdheim hat ein eigenes Modell des „gesellschaftlich Unbewussten“ entwickelt (siehe dazu Kapitel 4.2.2). 
der Gruppenanalyse weiterentwickelt, haben aber auch durch die Ausführungen von Erdheim eine spezifische Ausformung erfahren.

\subsubsection{Erich Fromm: Der Gesellschaftscharakter und das gesellschaftiche Unbewusste}

Fromm gehört zu jenen Analytikern, die in Auseinandersetzung mit dem Werk Freuds bereits sehr früh eine besondere Gewichtung des gesellschaftlichen Faktors vornahmen. Fromm ist der Erste, der dezidiert von einem ,gesellschaftlichen Unbewussten" spricht und ein Konzept von einem sozialtypischen Charakter entwickelt. Begrifflichkeiten, die sich auf die gesellschaftliche oder soziale Dimension des Unbewussten beziehen, finden sich auch bei anderen Psychoanalytikern, wie im Rahmen dieses Kapitels noch ausführlich beschrieben werden wird.

Im folgenden Abschnitt geht es darum, die Eigenständigkeit von Fromms Ansatz darzustellen und, wo für das Verständnis notwendig, auch auf Unterschiede zu den Arbeiten Freuds und seiner Zeitgenossen einzugehen. Der Abschnitt teilt sich in zwei Teile, in „Der Gesellschaftscharakter“ und „Das gesellschaftliche Unbewusste" und schließt mit weiterführenden Überlegungen, die bei der Auswertung der Interviews eine Rolle spielen werden.

\subsubsection{Der Gesellschaftscharakter}

Fromm sieht den Menschen nicht sekundär, sondern „primär als soziales Wesen“ (Fromm, 1990a, S. 39), das von Anbeginn von gesellschaftlichen Kräften, Zwängen und Strukturen bestimmt wird.

„Die ,geschichtlichen'Veränderungen, das heißt, die Evolution des Menschen, beruhen nicht auf Veränderungen seiner anatomischen oder physiologischen Struktur, sondern finden auf Grund psychischer Veränderungen statt, die sich bei der Anpassung an das gesellschaftliche System, in das ein Mensch hineingeboren wird, ergeben "(Fromm, 1990c, S. 20).

Die individuelle Charakterentwicklung ist mit dem Gesellschaftscharakter durch Wechselwirkungsprozesse verbunden und nur durch eine Verschiebung in der Wahrnehmung zu trennen, sodass ,die psychische Struktur, die wir als wirksam im gesellschaftlichen Prozess erkennen können, [...] selbst schon Produkt dieses Prozesses [ist]“"(Fromm, 1937, S. 23).

„Die sozio-biologische Form des Charakters bestimmt nicht nur die Bildung des individuellen Charakters, sondern auch des Gesellschafts-Charakters. Der Gesellschafts-Charakter enthält die, Matrix ' oder den ,Kern' der Charakterstruktur der meisten Mitglieder einer gesellschaftlichen Gruppe" (Fromm, 1990c, S. 23). 
Den Einwand, dass ein Baby oder Kleinkind mit der Gesellschaft als solcher ja erst viel später in Kontakt kommt, lässt Fromm nicht gelten, da die Eltern selbst durch kulturelle, ökonomische und ideologische Einflüsse geprägt sind.

„Wir verstehen deshalb die Charakterentwicklung in erster Linie als eine Antwort des Menschen auf die gesamte Konfiguration der Gesellschaft, deren Teil er ist, wobei diese Konfiguration ursprünglich durch die Familie vermittelt wird" (Fromm, 1990c, S. 104).

Fromm erkennt es als besondere Leistung der Freud'schen Theorie an, den Charakter nicht bloß als Bündel von Verhaltensmerkmalen aufzufassen, sondern als Ausdruck der Konflikthaftigkeit, in die der Mensch aufgrund seiner triebhaften und kulturellen Existenz gebracht wird. Im Gegensatz zu Freud ${ }^{51}$ sieht er den Charakter aber nicht nur als Ergebnis einer Fixierung auf bestimmte prägenitale und genitale Triebstrebungen, sondern auch als Ausdruck einer bestimmten Lebenshaltung an. Diese ist als Reaktion auf bestimmte Umweltkonstellationen und als Repräsentanz von Objektbeziehungen zu verstehen, die - und das ist wichtig - unbewusst bleiben.

Der Charakter verleiht Sicherheit in der Orientierung und wegen den mit anderen geteilten Erfahrungen und Vorstellungen auch die Sicherheit, Teil einer Gemeinschaft zu sein, was, wie später noch erläutert wird, ein Mittel der Angstreduktion ist.

„Der Charakter ist jene Form, in die menschliche Energie im Prozess der ,Sozialisation' (in der Bezogenheit zu anderen) und der ,Assimilierung' (in der Art und Weise der Aneignung von Dingen) kanalisiert wird. Der Charakter ist eigentlich der Ersatz für die fehlenden Instinkte " (Fromm, 1990c, S. 22).

Neben den Produktions- und Verteilungsmethoden einer Gesellschaft tragen auch die religiösen, politischen und philosophischen Ideen zur Genese des Gesellschaftscharakters bei (vgl. Fromm, 1955, S. 60 f.). So wird für Fromm exemplarisch der ,anale Charakter“ im Sinne einer spezifischen Struktur von Impulsen, Ängsten und Haltungen zum „kleinbürgerlichen Charakter“ oder in einem weiteren Rahmen zum „bürgerlichen Charakter“ (vgl. Fromm, 1937, S. 75 ff.). Das heißt, jede Gesellschaft bildet, entsprechend ihren Produktions- und Verteilungsmethoden und ihrer Organisation, einen ,sozial typischen Charakter" aus, den ihre Mitglieder teilen (vgl. Fromm, 1937, S. 77). Es handelt sich dabei nicht um einen bestimmten Durchschnittswert der Bevölkerung, sondern um charakterliche Tendenzen, die unter bestimmten Produktionsverhältnissen verstärkt hervortreten und diesen dienen.

51 Dieser Gegensatz ist in dieser Schärfe aus Freuds Schriften nicht abzuleiten. 
Die Funktion des „Gesellschaftscharakters“ oder auch „sozialen Charakters ${ }^{\text {‘.52 }}$ besteht nach Fromm darin, dass das Verhalten der Mitglieder einer Gesellschaft

„,nicht von der bewussten Entscheidung abhängt, ob sie sich nach dem gesellschaftlichen Modell richten wollen oder nicht, sondern dass sie so handeln wollen, wie sie handeln müssen ${ }^{53}$ und dass es ihnen gleichzeitig eine gewisse Befriedigung gewährt, wenn sie sich den Erfordernissen ihrer Kultur entsprechend verhalten " (Fromm, 1955, S. 60).

Nur so ist es verständlich, dass Menschen freiwillig Arbeiten erledigen, die ihnen aufgetragen werden, oder dass sie konsumieren, um glücklich zu sein. Der sozialtypische Charakter bestimmt genauer, welche Befriedigungen in materieller oder ideologischer Hinsicht für die Menschen möglich sind, um im Sinne der Gesellschaftsstruktur ihre Aufgaben zu erfüllen und ihre Energie zur Verfügung zu stellen. Dazu gehören auch Religionen und Ideologien mit entsprechenden Verboten und Geboten, die die Aufgabe haben, „die durch eine bestimmte Wirtschaftsweise bedingte Charakterstruktur $\mathrm{zu}$ verstärken und $\mathrm{zu}$ integrieren“ (Fromm, 1937, S. 89). So wird etwa die Konsumhaltung, die sich im letzten Jahrhundert in den westlichen Gesellschaften etabliert hat, zu einem Charakterzug und zu einer Matrix, die der Aufrechterhaltung des kapitalistischen Gesellschaftssystems dient.

Der Gesellschaftscharakter ist nach Fromm wie ein „Transmissionsriemen“ (Fromm, 1962, S. 89) zu verstehen, der die ökonomische Basis mit Ideen und Idealen verbindet und in beiderlei Richtungen wirkt (vgl. Fromm, 1962, S. 89 ff.). Wie sehr die Menschen durch die spezifischen Anforderungen einer Gesellschaft geformt werden, wie sehr ihre Bedürfnisse fremdbestimmt sind und wie sehr ihr Verhalten krankmachende Tendenzen zeigt, bleibt unbewusst; sie erleben ihre Anpassung daher als persönliche Freiheit.

„Der Gesellschafts-Charakter, der die Menschen veranlaßt, so zu handeln und zu denken, wie es der reibungslose Ablauf ihres gesellschaftlichen Lebens erfordert, ist nur das eine Verbindungsglied zwischen Gesellschaftsstruktur und Ideen. Das andere Verbindungsglied ist die Tatsache, daß eine jede Gesellschaft bestimmt, welche Gedanken und Gefühle ins Bewußtsein gelangen dürfen und welche unbewußt bleiben müssen. Genauso wie es einen Gesellschafts-Charakter gibt, gibt es auch ein ,gesellschaftliches Unbewußtes " ${ }^{54}$ (Fromm, 1962, S. 96).

52 Fromm verwendet beide Begriffe (Fromm, 1975, S. 190).

53 Hervorhebung durch Fromm.

54 Hervorhebung durch Fromm. 


\subsubsection{Das gesellschaftliche Unbewusste}

Fromm postuliert neben dem individuellen Unbewussten ein gesellschaftliches Unbewusstes.

An dieser Stelle ist es notwendig, darauf hinzuweisen, dass Fromm die Gesellschaft als solche nicht wie einen eigenen Organismus behandelt, wie das in manchen Konzepten des sozialen Unbewussten, die in den letzten Jahren und Jahrzehnten entwickelt wurden, diskutiert wird. Er präzisiert:

„Bisher habe ich immer vom Standpunkt der Gesellschaft aus argumentiert, von den Bedürfnissen einer gegebenen Gesellschaft und von ihrem Überlebensdrang. Ich möchte damit nicht behaupten, dass eine Gesellschaft denke oder sonst irgendetwas tue, doch hat ein soziales System seine eigene Logik und Dynamik und setzt zu seinem Funktionieren eine bestimmte Art des Verhaltens und Fühlens voraus" (Fromm, 1956, S. 107).

Das gesellschaftliche Unbewusste ist als ein von mehreren Mitgliedern geteiltes Unbewusstes zu verstehen, das neben bzw. mit dem persönlichen Unbewussten existiert und in seiner Funktion Konflikte vermeiden helfen soll.

„Bei diesen von der Allgemeinheit verdrängten Elementen handelt es sich um Inhalte, die den Mitgliedern der jeweiligen Gesellschaft nicht bewusst werden dürfen, wenn diese Gesellschaft mit ihren spezifischen Widersprüchen reibungslos funktionieren soll" (Fromm, 1962, S. 96).

Es kommt zu einer Auslese, „welche ${ }^{55}$ Erfahrungen ins Bewußtsein kommen dürfen. Dieser ,Filter' ist gesellschaftlicher Natur und besteht aus Sprache, Logik und Sitten (tabuisierte bzw. erlaubte Ideen und Impulse)“"(Fromm, 1990a, S. 81).

„Natürlich variieren die Inhalte des gesellschaftlichen Unbewußten entsprechend den vielen unterschiedlichen Formen der Gesellschaftsstruktur: Es handelt sich je nachdem um Aggressivität, Aufbegehren, Abhängigkeit, Einsamkeit, Kummer und Langeweile, um nur einige Impulse zu erwähnen. Der verdrängte Impuls muss unterdrückt bleiben und durch Ideologien ersetzt werden, die ihn verleugnen oder sein Gegenteil behaupten" (Fromm, 1965, S. 408).

Die Filter könnte man auch als Zensur verstehen, in deren Dienst die Verdrängung operiert. Sollte die Verdrängung aufgehoben werden, würde dies, wie Fromm ausführt, bestimmte Ängste verstärken. Auch in der Gewichtung der Ängste unterscheidet er sich von Freud.

55 Hervorhebung durch Fromm. 
„Es ist die Angst vor der Isolation und vor der Ächtung, und nicht die ,Kastrationsangst', die die Menschen veranlaßt, das zu verdrängen, was tabu ist, weil dessen Gewahrwerden bedeuten würde, daß man andersartig, abgesondert und daher von den anderen geächtet ist [...] Deshalb zieht die Angst vor der Ächtung auch die Angst vor dem Identitätsverlust nach sich, und die Verbindung beider Ängste übt auf den Menschen die stärkste Wirkung aus " (Fromm, 1962, S. 119f.).

Die Inhalte des Unbewussten sind nach Fromm frei von einer Wertung.

„Der Inhalt des Unbewußten ist daher weder gut noch böse, weder rational noch irrational; er ist alles, was menschlich ist. Das Unbewußte ist der ganze Mensch außer dem Teil von ihm, welcher seiner Gesellschaft entspricht ${ }^{56}$ "(Fromm, 1962, S. 121).

Das gesellschaftliche Unbewusste hat, wie Fromm betont, nichts mit dem kollektiven Unbewussten von Jung zu tun, da es ihm selbst nicht um von allen Menschen geteilte archaische Inhalte geht, sondern um Verdrängungsprozesse im Dienste von Gesellschaftsstrukturen, die damit deren Bestehen gewährleisten (vgl. Fromm, 1990a, S. 81 f.). In einer Fußnote präzisiert Fromm den Unterschied dahingehend, dass das ,kollektive Unbewusste“ nach Jung sich auf die universale Psyche bezieht, von der ein großer Teil nicht bewusst werden kann.

„Der Begriff des gesellschaftlichen Unbewußten geht dagegen von der Verdrängung innerhalb einer Gesellschaft aus und bezieht sich auf den spezifischen Teil menschlicher Erfahrung, welchen eine bestimmte Gesellschaft am Bewußtwerden hindert; es handelt sich dabei um den Teil der Humanität im Menschen, den seine Gesellschaft ihm entfremdet hat; das gesellschaftliche Unbewußte ist der von der Gesellschaft verdrängte Teil der universalen Psyche" (Fromm, 1962, S. 112).

Entfremdung ist für Fromm

„, eine besondere Form der Unbewußtheit [...] Sie ist ein Pseudobewusstsein von Erleben, bei der sich der entfremdete Mensch hinsichtlich seines Erlebens selbst betrügt. Er ist nicht mit seinem Erleben in Kontakt, sondern zum Beispiel nur mit seinem Denken, mit seinem Götzen " (Fromm, 1959, S. 132).

Der Prozess der Entfremdung und der „Götzendienst“ beschreiben nach Fromm das gleiche Phänomen. „Hegel und Marx sprachen von Entfremdung, die Propheten des Alten Testaments von Götzendienst" (Fromm, 1959, S. 121). Dabei wird das eigene Erleben auf etwas oder jemanden projiziert, mit dem Effekt, dass man mit sich selbst, mit seinem eigenen Sein über diesen Umweg in Kontakt kommt. Fanatismus, Nationalismus oder Antisemitismus wie die Unterwerfung

56 Hervorhebung durch Fromm. 
unter bestimmte Ideale sind ebenso als Phänomene der Entfremdung zu verstehen. Sie produzieren intensive Gefühle von Leidenschaft und Intensität (vgl. Fromm, 1959, S. 128 f.).

Das Verhältnis von bewusst und unbewusst wird in zweifacher Hinsicht als veränderbar angesehen: erstens, was die Gesellschaftsstrukturen betrifft, die sich in ihren sozioökonomischen Grundlagen unterscheiden und verändern, und zweitens was die individuellen Unterschiede und Zugangsweisen zur Realität betrifft (vgl. Fromm, 1990a, S. 83). In Meditationszuständen, in Träumen oder unter Drogeneinfluss zeigen sich andere Zugänge zur Welt, die sich von den sogenannten bewussten Zugängen unterscheiden. Unbewusstes bewusst zu machen im Freud'schen Sinn gilt als Ziel sowohl für das individuelle als auch für das gesellschaftliche Unbewusste. Fromm bringt diesen Prozess mit der Suche nach Wahrheit in Verbindung.

„Das Gewahrwerden des Unbewußten wird zu einem wesentlichen Element der Wahrheitssuche; Erziehung wird zum Prozeß der Desillusionierung, der ,Rücknahme der Täuschung '(de-ception) "(Fromm, 1990a, S. 84 f.).

Freud und Marx unterscheiden sich nach Fromm hinsichtlich der Einschätzung der Rolle der Verdrängung im historischen Prozess. Während für Freud der Zivilisationsprozess unweigerlich mit einer Zunahme an Verdrängung verbunden ist, gibt es für Marx in einer Gesellschaft, die frei von Ausbeutung und Klassengegensätzen ist, keine Verdrängung (vgl. Fromm, 1962, S. 124). Fromm selbst teilt diese optimistische Sicht nicht.

Als Wirkfaktoren für die Aufhebung von Verdrängung sieht Fromm positive Zukunftsvorstellungen an, weil sie dabei helfen, eine realitätsgerechtere Sicht zu entwickeln (vgl. Fromm, 1962, S. 122). Aber auch Stille, Ruhe und Konzentration werden genannt. Kritisch beurteilt Fromm den therapeutischen Wirkfaktor, ob es sich nun um Einzel- oder Gruppentherapien handelt.

„Die heute so modern gewordene Idee, sein Unbewußtes dadurch zu entdecken, daß man, ganz frei und offen 'in einer Gruppe darüber spricht, scheint mir auf einer Illusion aufzubauen " (Fromm, 1990a, S. 91).

Aus der klinischen Praxis weiß man, dass Reden und Denken in den Dienst der Abwehr gestellt werden können. Fromm hebt dagegen die Bedeutung des Körpers hervor, der einen Zugang zum Unbewussten schaffen kann.

„Der Körper ist ,ein Symbol der Seele‘: Die Gestalt des Körpers, die Haltung, die Art des Gehens, die Gesten, der Gesichtsausdruck, das Atmen und die Sprechweiseall dies sagt viel, ja mehr über das Unbewußte eines Menschen aus als fast alle anderen Informationen, die herkömmlicherweise im psychoanalytischen Prozess gebraucht werden " (Fromm, 1990a, S. 96). 
Fromm äußert sich in wertschätzender Weise über die Arbeiten Reichs, der die Herstellung der orgastischen Potenz, die Auflösung der Spannungen, die sich als Ausdruck von Verdrängung und Widerstand in einem körperlichen Panzer zeigen, als wesentlich erachtete.

„Seine Auffassung der genitalen Potenz sprengte die Grenzen des Unlust-Lust-Prinzips. Für ihn ist die genitale Potenz die Reaktion eines Menschen, der nicht verdrängt und abwehrt, die Reaktion eines das Leben bejahenden und sich des Lebens erfreuenden freien menschlichen Wesens" (Fromm, 1990b, S. 121).

Ihrer beider Fokus, was das Wirkverhältnis zwischen Individuum und Gesellschaft betrifft, ist jedoch unterschiedlich:

„Während Reich sich besonders auf die Beziehung zwischen Sexualmoral, Verdrängung und Gesellschaft konzentrierte, stand im Mittelpunkt meines Interesses der ,Gesellschafts-Charakter; also jene ,Charaktermatrix; die von den Mitgliedern einer Gesellschaft oder gesellschaftlichen Klasse geteilt wird und durch die allgemeine menschliche Energie in eine spezifisch menschliche Energie verwandelt wird, die für den Bestand einer gegebenen Gesellschaft notwendig ist" (Fromm, 1990a, S. 92 f.).

Bei aller Wirkkraft des Sozialen verweist Fromm immer wieder, wie schon eingangs dargestellt, auf das Zusammenspiel zwischen der Naturhaftigkeit des Menschen, seinen physiologischen Trieben und den ,historischen sich im gesellschaftlichen Prozess entwickelnden psychischen Impulse[n]" (Fromm, 1937, S. 59), die die Bedingungen seiner Existenz ausmachen. Dieses Zusammenwirken bestimmt, wie der Mensch auf äußere Bedingungen reagiert. Dazu gehört auch, dass der Gesellschaftscharakter nach Fromm seine Macht verliert, wenn er den menschlichen Bedürfnissen zu sehr widerspricht. „So sehr der GesellschaftsCharakter in Perioden sozio-ökonomischer Stabilität der Zement der Gesellschaft ist, so wird er in Zeiten drastischer Änderungen zu Dynamit" (Fromm, 1990c, S. 24). Fromm führt als Beispiel dafür an, wie das Kleinbürgertum durch die Verschlechterung der ökonomischen Bedingungen die für es typischen Bedürfnisse nach Arbeit, Sparen und Selbstständigkeit nicht mehr befriedigen konnte. Die Möglichkeiten ideologischer Beeinflussung sind nach Meinung Fromms dabei beschränkt, weil die realen Lebensverhältnisse größere Wirkung zeigen (vgl. Fromm, 1937, S. 84 f.). Fromm schreibt:

„Meiner Meinung nach ist das kritische Verständnis und Gewahrsein der Fiktionen in den bei uns gängigen gesellschaftlichen Verhaltensmustern eine unverzichtbare Voraussetzung für das volle Bewusstsein des dissoziierten Teils in einem anderen Menschen" (Fromm, 1959, S. 150). 
Gesellschaftliches Unbewusstes bewusst zu machen, bedeutet daher auch für die Psychoanalyse, das Gegebene, Bekannte, Vertraute zu hinterfragen und die „Pathologie der Normalität“" (Fromm, 1990a, S. 37) zu erforschen.

Das ist eine wesentliche Zielsetzung der vorliegenden Arbeit.

\subsubsection{Mario Erdheim: Das gesellschaftlich Unbewusste}

Erdheim (2013) unterscheidet den Begriff des „gesellschaftlich Unbewussten“ vom „gesellschaftlichen Unbewussten“, weil Letzteres die Vorstellung der Gesellschaft als Organismus evozieren könnte, der selber ein Unbewusstes produziert.
„Ersteres bedeutet, dass das Unbewusste ein individuelles Phänomen ist, das meh- reren Individuen gemeinsam ist; Letzteres bedeutet, dass das Unbewusste ein gesell- schaftliches Phänomen ist, wobei angenommen wird, dass die Gesellschaft über ein Unbewusstes verfügt (etwa im Sinne eines kollektiven Unbewussten). Eine weitere Nuance: das gesellschaftlich Unbewusste ist ein (immer individuelles) Unbewusstes, das gesellschaftlich produziert wird, nämlich mit Hilfe von Institutionen, während das gesellschaftliche Unbewusste quasi eine überindividuelle Substanz postuliert, die aber nirgends lokalisiert werden kann "(Erdheim, 2013, S 1026).

In diesem Sinn sind die Träger des gesellschaftlich Unbewussten immer Individuen. Am Beispiel des Phantasmas, eines Vorstellungskomplexes, der von verschiedenen Individuen geteilt und dessen Wirklichkeitscharakter von ihnen beansprucht wird, zeigt Erdheim auf, wie zum Beispiel die Phantasmen der Hexe oder des Teufels in Zeiten des absoluten Herrschaftsanspruchs von Kirche und Staat dazu verwendet wurden, um Machtverhältnisse zu festigen. Mittels Spaltung, eines Mechanismus der Unbewusstmachung, werden die Herrschenden geschont und wird die Aggression, die auf sie gerichtet werden könnte, auf Feindbilder abgelenkt; darauf wird noch weiter unten genauer eingegangen.

„Die Gestalt des Teufels, dessen Macht sich angeblich immer mehr ausdehnte, spiegelt die vom Unbewussten verzerrten Züge des absoluten Fürsten. Da man aber den Teufel statt des Königs bekämpfte und nicht den Adel, sondern die Hexen jagte, dienten diese Ersatzbildungen letztlich der neuen Staatsform und der Zementierung ihrer Macht" (Erdheim, 2013, S. 1027).

Die Funktion des Phantasmas besteht zum einen also darin, dass es „im Sinne einer Gegenbesetzung etwas nicht zu Bewusstsein kommen lässt, es im Unbewussten behält und so auch dem Verständnis entzieht" (Erdheim, 2013).

Mit dem Verweis auf Freuds Arbeit „Totem und Tabu“ schreibt Erdheim Phantasma und Totem ähnliche Funktionen zu. Der von den Söhnen getötete Vater der Urhorde wird zum Totem erhoben und in seiner Macht noch erhöht. 
Die Söhne hassten den Vater nicht nur, sie liebten ihn auch. Unbewusst gemacht werden also Hass und Mord. Das Totem hat für Erdheim daher eine ähnliche Funktion wie das Phantasma (vgl. Erdheim, 2013, S. 1030), es geht um „die Verdrängung eines für die Gesellschaft wichtigen Ereignisses“ (Erdheim, 2013, S. 1029), um seine Unbewusstmachung, und gleichzeitig um die Legitimierung der neuen oder alten Machthaber.

So kann man auch verstehen, wie sich Herrschaftsverhältnisse über Generationen hinweg etablieren, aber auch, wie Traumen weitergegeben werden. Innerpsychisch wird die Ambivalenz begleitet von identifikatorischen Prozessen. Obwohl man den Vater bekämpft, möchte man, vereinfacht ausgedrückt, auch so sein wie er und seine Macht ausüben, „bewusst sind lediglich die Ablehnung, die Kritik sowie der Kampf gegen die Herrschaft" (Erdheim, 2013, S. 1031).

Mit Herrschaft ist jedoch mehr als die Machtausübung in einer kleinen sozialen Einheit gemeint; es geht um die Strukturierung des gesellschaftlichen und politischen Zusammenlebens, um Geschlechterverhältnisse, Arbeitsverhältnisse, ökonomische Bedingungen.

In kritischer Auseinandersetzung mit Devereux’ Begriff des „ethnisch Unbewussten", der in Bezug auf das, was von einer spezifischen Kultur an Verdrängungsleistung gefordert wird (vgl. Erdheim, 2013, S. 1027 f.), eine gesellschaftliche Homogenität suggeriert, bezieht sich Erdheim auf Bernfelds Definition des „sozialen Ortes“ (Bernfeld, 1974a), das heißt einer spezifischen Gebundenheit des gesellschaftlich Unbewussten an die soziale Stellung des Individuums. ${ }^{57}$

„Man kann die Fragestellung nach dem historischen Aspekt und nach der Milieuprägung eines seelischen Vorgangs als den Gesichtspunkt des, sozialen Orts 'zusammenfassen und hervorheben. Man sagt damit gewiss nichts Neues, sondern der soziale Ort ist ein Sektor dessen, was die Psychoanalyse als Realität bezeichnet. Gegebenenfalls wird diese Realität verinnerlicht und der soziale Ort wird dadurch zu einem Moment am Über-Ich" (Bernfeld, 1974a, S. 210).

Das bedeutet, dass je nach Gruppenzugehörigkeit und Erfahrung bei den einzelnen Menschen, in unserem Fall zum Beispiel bei den Nutznießern der Finanzkrise oder den Geschädigten, andere Aspekte der sozialen Realität unbewusst gemacht werden.

57 Siehe dazu die Ausführungen zu Bernfelds Theorie des sozialen Orts in Kapitel 5.1.2. Parin argumentiert ähnlich: „,Macht- und Produktionsverhältnisse mit allen von ihnen abgeleiteten Institutionen, Regel- und Wertsystemen bedürfen einer Erschließung und Enthüllung - vergleichbar der Arbeit, welche die Psychoanalyse ehedem mit der psychischen Instanz ,Unbewußt‘ geleistet hat“ (Parin, 1975, S. 518 f.). 
Ausgehend von Freuds „Die Zukunft einer Illusion“ (S. Freud, 1927c) lenkt Erdheim den Blick auf Hass und Gewalt, die als Reaktion auf Unterdrückung und Ungerechtigkeit in den Individuen entstehen.

„In diesen Prozessen wird die entlastende psychohygienische Funktion der Fremden wirksam: ich hasse nicht meine Nächsten, die mir die Erfüllung meiner Wünsche versagen, sondern ich hasse die Fremden, die als Feinde erlebt werden, die nicht in meine Umgebung gehören. Der Hass und die Feindseligkeit gegen die eigene Gesellschaft und Kultur ist ein kaum auszuhaltendes Gefühl und wird oft auf eine ähnliche Weise tabuisiert wie der Hass auf die eigene Familie"(Erdheim, 2013, S. 1033).

Soziale Gruppen oder Einzelpersonen übernehmen die Funktion des Sündenbocks, auf den die Aggressionen verschoben und damit die wahren Verursacher unkenntlich gemacht werden.

Erdheim sieht die grundsätzliche Spannung zwischen Individuum und Gesellschaft mit der Neuregelung verbunden, die in Freuds „Totem und Tabu“ auf den Vatermord folgt. Dieser Vatermord und das Inzesttabu zerstörten das Hordenprinzip und ,ersetzten es durch die antagonistische Dualität von Familie und Kultur", die unterschiedlichen Prinzipien gehorchen (Erdheim, 2013, S. 1036).

In der Adoleszenz besteht - anders als in der ödipalen Phase, wo das Liebesobjekt aufgegeben werden muss - die Möglichkeit, eine reale libidinöse Befriedigung zu erleben, sich aus der Familie zu lösen und neue Erfahrungen zu machen. Die zentrifugalen Kräfte der Gesellschaft sind dafür verantwortlich, dass sich die jungen Menschen mit anderen Individuen libidinös verbinden können, während die zentripetalen Kräfte der Familie zu Intimität und Abschottung führen (vgl. Erdheim, 2013, S. 1038 f.). In einem Extrem produzieren die zentripetalen Kräfte daher Wissensverlust und Angst vor dem Fremden, gepaart mit Ausbrüchen von Gewalt, die zentrifugalen Kräfte dagegen einen Bindungsverlust.

„Die Jugend, deren Bezug zur Familie völlig zerstört wird, verwandelt sich in ein willfähriges Instrument der Machthaber, die Unwissenheit, Angst und Hass der Jugendlichen in den Dienst ihrer Interessen stellen “ (Erdheim, 2013, S. 1039).

Was nun die Produktion des Unbewussten betrifft, konstatiert Erdheim Folgendes:

„[...] das gesellschaftlich Unbewusste der Familie muss alles, was die zentrifugale Bewegung der Kultur weiter befördert, verdrängen, und die Kultur muss ihrerseits alles, was die zentripetale Bewegung begünstigen würde, unbewusst machen" (Erdheim, 2013, S. 1038). 
Dieses Konzept des gesellschaftlich Unbewussten ermöglicht einen Blick auf das Individuum und seinen „sozialen Ort“, der in der Definition von Bernfeld die Geschichtlichkeit einschließt und auch das Unbewusste in der Wissensproduktion erhellt.

„Ohne diesen Begriff des Unbewussten tendieren die Kulturwissenschaften dazu, gesellschaftliche Verdrängungsprozesse ebenso wie die Abspaltungen zu reproduzieren statt sie sichtbar zu machen " (Erdheim, 2013, S. 1027).

Ich möchte diese Auffassung meiner Arbeit zugrunde legen, wenn es darum geht, die Auswirkungen der Finanzkrise auf die Psyche zu untersuchen. Erdheim schreibt:

„In der Konsumgesellschaft kommt noch ein weiterer Hass produzierender Faktor ins Spiel. Während in vorkapitalistischen Zeiten Frömmigkeit und asketische Übungen, oft genug auch wirtschaftliche Not und soziale Katastrophen Bescheidenheit zur Tugend machten, löste die kapitalistische Kultur eine Wunschexplosion und damit auch eine Dynamik aus, die das Wünschen zu einem wesentlichen Motor der Entwicklung machte. Das Wünschen wurde zu einer Art Produktivkraft, auf die man nicht ohne Weiteres wird verzichten können "(Erdheim, 2013, S. 1033 f.).

In der Darstellung der analytisch-sozialpsychologischen Matrix in Kapitel 6 wird versucht, Licht in das Dunkel der Fragen zu bringen, wer von der Finanz- und der ökonomischen Krise profitiert, wer ihre Opfer sind und welche Kompromissund Ersatzbildungen entstehen, um Herrschaftsverhältnisse zu festigen.

\subsubsection{Das soziale Unbewusste: Die Entwicklung des Konzepts mit besonderem Schwerpunkt auf der Gruppenanalyse}

Hopper und Weinberg beschreiben in der Einleitung zu ihrem Buch „The Social Unconscious in Persons, Groups and Societies“ (2011) die Entwicklung des Konzepts des sozialen Unbewussten ausgehend von den Modellen des Unbewussten bei Freud. Bereits in den 20er- und 30er-Jahren des letzten Jahrhunderts haben Freuds Weggefährten diese Konzeptionen durch die gesellschaftlich beeinflussten Dimensionen menschlichen Denkens, Handelns und Fühlens erweitert. Dabei trifft man auf durchaus divergierende Ansätze und Begrifflichkeiten, wie sie in den Arbeiten von Bernfeld, Foulkes, Fromm und Jung auftauchen. So hat der Gruppenanalytiker Siegmund Heinrich Fuchs, später Foulkes ${ }^{58}$, darauf

58 Er wurde als Siegmund Heinrich Fuchs in Deutschland geboren und emigrierte 1933 nach England. 
hingewiesen, dass es neben den unbewussten Aspekten, die bereits Freud beschrieben hat, auch ein soziales Unbewusstes gebe, das internalisiert zur Wirkung komme. Foulkes arbeitete zu jener Zeit in Frankfurt und kannte die Arbeiten von Bernfeld, Fromm, Fromm-Reichmann und Horney. Er beschreibt das soziale Unbewusste folgendermaßen:

"[...] a totally different area of which the individual is equally unaware [...] [and is] [...] as much compelled and moulded by these colossal forces as by his own id, and defends himself as strongly against their recognition without being aware of it, but in quite different ways and modes. One might speak of a social or interpersonal unconscious " (Foulkes, zitiert nach Hopper \& Weinberg, 2011, S. xxvi).

In anderen seiner Arbeiten taucht dafür auch der Begriff ,interpersonal unconscious" auf (vgl. Hopper \& Weinberg, 2011, S. xxvi). Die Autoren beklagen an dieser Stelle auch, dass Foulkes dieses Konzept nicht weiter elaboriert und es auch nicht in einen therapeutischen Kontext gestellt hat.

Zentral ist bei Foulkes die enge Verflochtenheit von Individuum und Gesellschaft, die nur durch eine Verschiebung der Perspektive in der Wahrnehmung getrennt werden kann.

"Foulkes believed that the body, mind, and society are completely interdependent and intertwined with one another. Thus, ,Individuals " and their, Groups' are abstractions constructed by the observer of them, and a matter of the figure and ground of an observer's perception of them" (Hopper \& Weinberg, 2011, S. xxvi f.).

Ähnliche Begrifflichkeiten findet man bei Moreno, dem Begründer der Soziometrie, der für die wechselseitigen Beeinflussungen von Gesellschaft und Individuen die Begriffe „,co-unconscious“ und ,inter-personal unconscious“ benützte (Hopper \& Weinberg, 2011, S. xxvii).

In den 1960er-Jahren begannen einzelne Gruppenanalytiker in Opposition zum Trend jener Zeit ihre Skepsis gegenüber dem Einfluss von sozialen Faktoren auf das Individuum abzulegen.

"[...] they used a model of the mind that was developed by Winnicott and various members of the Group of Independent Psychoanalysts who developed the British object relations school of thought" (Hopper \& Weinberg, 2011, S. xxviii).

Hopper, Soziologe, Psychoanalytiker und Gruppenanalytiker, entwickelte, basierend auf Winnicotts Objektbeziehungstheorie und in Auseinandersetzung mit Foulkes und Horney, sein eigenes Modell des sozialen Unbewussten, in das er anthropologische, sprachwissenschaftliche, soziologische und psychoanalytische Weiterentwicklungen integrierte. 
"Essentially, Hopper accepted the view that the 'social unconscious' refers to the fact that people are likely to be unconscious of social factors and forces, but noted that the concept is also used to refer to the social factors and forces of which they are unconscious [...]" (Hopper \& Weinberg, 2011, S. xxix).

Wie Hopper beschreibt, ist es von Bedeutung, worauf man sich beim Gebrauch des Begriffs des sozialen Unbewussten bezieht, auf das Individuum oder auf ein soziales System - genauer gesagt, auf die internalisierten Faktoren eine äußeren Welt, derer man sich nicht bewusst ist bzw. auf Aspekte der äußeren Welt, derer sich zumindest drei Personen nicht bewusst sind. Damit kommt ins Spiel, dass das soziale Unbewusste die Repräsentanz eines interpersonellen Geschehens ist, das sich auf die äußere Welt bezieht.

"More specifically, Hopper defined the social unconscious in terms of the existence of social, cultural, and communicational 'arrangements' of which people are 'unaware'. [...] being unaware [...] is the relational process involving three or more persons, and is never merely a matter of a single individual's knowledge of the external social world" (Hopper \& Weinberg, 2011, S. xxx).

Verständlich ist in diesem Zusammenhang, dass nicht alle Faktoren der äußeren Welt zugleich Faktoren der sozialen Welt sein müssen, umgekehrt gilt dies jedoch schon. Die Autoren führen eine Reihe von Beispielen an, wie den Spracherwerb, das Entstehen von Bindung oder die Entwicklung von Empathie und Kreativität, die verdeutlichen, wie sehr das Individuum von Beginn an auf den Austausch mit der äußeren Welt angewiesen ist, dass diese äußere Welt nicht nur aus Projektionen besteht, sondern realen Einfluss auf die Menschen hat. In Internalisierungen sind daher sowohl gute als auch weniger gute Objekte vorhanden.

"It is likely that the internalization of social objects is, to some degree, always associated with a sense of absence, loss, and/or separation from objects on whom subjects are dependent" (Hopper \& Weinberg, 2011, S. xxxiv).

Genauso wie das Individuum sich gegenüber schmerzhaften, peinlichen und ängstigenden Erfahrungen - die, wie die Autoren sagen, biologisch, das heißt triebhaft, motiviert sind - schützen muss, gilt das auch für unangenehme Wahrnehmungen und Erfahrungen, die in Auseinandersetzung mit der sozialen Welt entstehen.

"In terms of this perspective, the social unconscious is maintained through a series of protective defences against the experience of painful anxieties that would follow from the recognition of certain social phenomena: social objects are not perceived (not 'known'), and if perceived, not acknowledged ('denied'), and if acknowledged, 
not taken as problematic ('given'), not considered with an optimal degree of detachment and objectivity ('conviction')" (Hopper \& Weinberg, 2011, S. xxxivf.).

Es wird in dieser Arbeit, wenn es um die Analyse der Interviews geht, ein spezieller Fokus darauf gelegt werden, inwiefern Abwehrvorgänge wie Verleugnung, Spaltung und Verdrängung bei der Auseinandersetzung mit der Finanzkrise und ihren Folgen als Gruppenerfahrung eine Rolle spielen.

Hopper und Weinberg gehen in ihren Ausführungen auch auf Differenzierungen ihres Konzepts ein, wie zum Beispiel jenes eines kulturellen Unbewussten, „cultural unconscious“, indem auf den Einfluss einer speziellen Kultur mit ihren Werten auf Internalisierungsprozesse hingewiesen wird (vgl. Hopper \& Weinberg, 2011, S. xxxiii f.).

In anderen Konzepten wird in Anlehnung an Fromm die Repräsentation von Herrschaftsstrukturen oder ökonomischen Strukturen im Unbewussten der Individuen in den Vordergrund gerückt. Fromms Konzept des gesellschaftlichen Unbewussten und des Gesellschaftscharakters wurde im Kapitel 4.2.1 dargestellt. In Abgrenzung zu Weinberg, der eine bessere Anwendbarkeit des Konzepts im Rahmen der empirischen Forschung wünscht, erachtet es Hopper als nicht sinnvoll, die einzelnen Elemente des sozialen Unbewussten aufzulisten.

"No list of the so-called contents of the societal social systems can be complete. For example: with respect to societal social systems, it is necessary to include language and communication systems in general, regardless of what is being communicated. Especially important are aspects of family structure, class and status group formations, ethnicity and classically classification, gender and social roles, and age grades, as well as the basic beliefs and mores of a society" (Hopper \& Weinberg, 2011, S. xxxix).

Eine Möglichkeit, das soziale Unbewusste zu untersuchen, ist die Gruppenanalyse. "The recognition of the validity of both the social fact and the psychic act is the basis of group analysis" (Hopper \& Weinberg, 2011, S. xl).

In der Gruppenanalyse wird auch die Hypothese diskutiert, ob es so etwas wie eine Gruppenpsyche (,group mind“) gebe. Die Autoren beziehen sich auf Foulkes, der behauptet, dass die freien Assoziationen im Rahmen einer Gruppe, die sich zur Therapie trifft, auch als Ausdruck einer bestimmten sozialen Realität gesehen werden muss.

"In other words, the mind of a particular group is a part of the mind of the society and/or of the minds of its intermediate institutions and organizations in which the group exists" (Hopper \& Weinberg, 2011, S. xl). 
Hopper hält es dagegen nicht für sinnvoll, von einem „group mind“ auszugehen. Gäbe es so etwas, müsste man sozialen Systemen auch ein Gehirn attestieren. Der zweite Grund für seinen Vorbehalt ist der, dass er sich, so wie auch andere, kritisch gegenüber dem Austausch von Begriffen äußert, die einerseits individuelle Besonderheiten und andererseits Besonderheiten von Gruppen beschreiben. Als Beispiel führt er die Begriffe „phallische“ oder „narzisstische Gesellschaft“ oder umgekehrt „,bürokratische Persönlichkeit“ oder „effiziente Persönlichkeit“ an (vgl. Hopper \& Weinberg, 2011, S. xlii, S. xIii). Eine solche Übertragung von Begriffen impliziert den Charakter von Institutionen oder Gruppen als lebendige Organismen, wie man ihn aus frühen Gesellschaftsmodellen kennt. „The notion of the ,mind" of the social system is really based on the metaphor that social systems are like people“" (Hopper \& Weinberg, 2011, S. xlii).

Problematisch an Gleichsetzungen von Individuum und Gruppe ist weiter, dass sie gesellschaftsstabilisierenden Charakter haben und weniger ein Änderungspotenzial in den Vordergrund rücken. Hopper führt nur eine einzige Ausnahme an, bei der er eine Gleichsetzung von Individuen und sozialen Systemen für gerechtfertigt hält. Diese bezieht sich auf traumatisierte soziale Systeme.

"[...] the social systems of wounded people also regress, for example, from complexity to simplicity: societies become like groups and groups become like their individual members. The structures of systems in which their participants have been traumatised are likely to weaken and collapse, and the participants in these systems are likely to become 'lost', not only in translation, but also in their interpersonal relationships, and in their commitment to shared values and norms" (Hopper \& Weinberg, 2011, S. xliiif.).

In traumatisierten Gesellschaften tendieren die Menschen dazu, ihre eigenen Erfahrungen, aber auch jene vorheriger Generationen unbewusst zu wiederholen und sich in einer Art Selbstschutz mit den Erfahrungen anderer zu verbinden (vgl. Hopper \& Weinberg, 2011, S. xliv).

Für Weinberg bilden Traumatisierungen das Herzstück aller Gesellschaften und spielen bei ihm daher keine gesonderte Rolle. Traumatisierungen eignen sich also nicht als Abgrenzungskriterium. Auch in Bezug auf die Frage, ob es so etwas wie eine Gruppenpsyche, ,group mind“, gibt, weicht er von Hoppers Ausführungen $a b$.

„Weinberg believes that in order to have a mind, a social system does not need to have a brain. [...] the flow of energy and information between the members of the social system is the mind of the social system. Thus, the mind of the social system exists within the transitional space that is co-created by all the members of the system, and is a function of their interpersonal relations and of their capacity for 'we-centric' space” (Hopper \& Weinberg, 2011, S. xlivf.). 
Für ihn ist der Begriff „social mind“ nicht nur ein Synonym für Kultur und/oder Organisationsstruktur eines sozialen Systems, sondern eine essenzielle Eigenschaft (vgl. Hopper \& Weinberg, 2011, S. xlv).

In den frühen 1970er-Jahren ersetzte Foulkes das Konzept des ,group mind“ durch das Konzept der „Matrix ${ }^{\text {“59 }}$, das auf eine Homologie von Gruppe und Individuum verzichtete.

"[...] the matrix refers to what sociologists called the 'organization' of a social system or the 'social organization'. The organization of the social system is multidimensional, and it is possible to consider many properties of it, such as patterns of interaction, normation, communication, and so on" (Hopper \& Weinberg, 2011, S. $x l v)$.

Während die „dynamic matrix“ einer Gruppe vorwiegend auf den Kommunikationsstrukturen basiert, basiert die „foundation matrix“ nicht nur auf eben diesen, sondern auch auf anderen Beziehungsstrukturen, Interaktionen und Wertvorstellungen ihrer Mitglieder. Beide Matrices sind in Veränderung begriffen, die „foundation matrix“ entwickelt sich jedoch über Generationen hinweg (vgl. Hopper \& Weinberg, 2011, S. xlvi f.).

$\mathrm{Zu}$ Recht beklagt Hopper, dass von Gruppenanalytikern der Tatsache wenig Beachtung geschenkt wurde, welchen Einfluss technologische und wirtschaftliche Veränderungen auf diese Matrices haben, wenn man bedenkt, wie zum Beispiel soziale Medien das Aufeinander-Bezogensein von Individuum und Gruppen verändert haben, indem etwa bei Facebook Einsamkeit und virtuelle Verbundenheit dieses Verhältnis neu bestimmen (vgl. Hopper \& Weinberg, 2011, S. xlvii).

Die Autoren gehen am Ende ihrer Einleitung noch auf den Unterschied zwischen dem sozialen Unbewussten und dem „kollektiven Unbewussten“ bei Jung ein. Neben dem individuellen Unbewussten teilen Menschen nach Jung auch ein kollektives Unbewusstes, das Archetypen enthält, Urbilder, die ein Erbe der Menschheit sind und in denen sich charakteristische Lebenserfahrungen widerspiegeln. Mythen werden als Ausdruck von frühen essenziellen kollektiven Erfahrungen der Menschheit angesehen.

59 „Foulkes used the term 'dynamic matrix' in reference to the social organization of the group, and the term 'foundation matrix' in reference to the social organization of the society. He did not have a concept for denoting the matrix of social systems that were larger and more complex than groups, but smaller and more simple than societies, such as organizations; neither did he have a concept for denoting the matrix of a family, which is not quite a group in the sense that all families are groups, but not all groups are families" (Hopper \& Weinberg, 2011, S. xlvi). 
"Jung assumed that mental life and biological life are governed by the principle that ontogeny recapitulates phylogeny. Thus, the great phenotypical-diversity is underpinned by a much smaller number of basic, innate, elemental archetypes and images of them. These primal fantasies predispose human beings to produce the same or similar mythical ideas and other collective phenomena over and over again" (Hopper \& Weinberg, 2011, S. xlix).

Von manchen Jungianern wird der Begriff des „shared unconscious“ verwendet, um die gemeinsame Konstruktion von sozialer Realität mit geteilten Ängsten und Abwehrmechanismen hervorzuheben. Andere wiederum betonen das ,cultural ingraining“, wenn sie darauf hinweisen, dass in unterschiedlichen Kulturen Archetypen ihre spezielle Ausformung erhalten. Wieder andere weisen mit dem Konzept des ,cultural complex“ darauf hin, dass Werte, Normen und Glaubenssätze unbewusst über die Generationen einer Kultur weitergegeben werden. In Abgrenzung dazu greift das Konzept des sozialen Unbewussten auf keinen kosmischen Urgrund zurück, in dem diese Erfahrungen wurzeln; es wird auch nicht von einem gemeinsamen Erbe gesprochen,

"the social unconscious is based on true transgenerational cultural inheritance through a variety of institutions such as education, child-rearing practices, and socialization more generally" (Hopper \& Weinberg, 2011, S. lf.).

Am Ende der Einführung fassen Hopper und Weinberg einige Argumente zusammen, warum das Konzept des sozialen Unbewussten auf so wenig Interesse bzw. großen Widerstand stößt. Es schmälert die eigene Vorstellung von Grandiosität, Omnipotenz und Allwissenheit. Die Nichtanerkennung des sozialen Unbewussten ist auch ein Schutz und damit ein Regulationsmechanismus für Ängste, die mit der eigenen Hilf- und Machtlosigkeit verbunden sein könnten. Diese gründen auf ganz frühen Abhängigkeitserfahrungen, auf der Tatsache, dass wir auf einen Austausch mit der Umgebung - beginnend im Mutterleib - angewiesen sind.

In diesem Zusammenhang müsste man auch die Vorstellung von einem freien Willen infrage stellen. Dazu kommen der in den westlichen Gesellschaften gelebte Individualismus und das Faktum, dass viele Psychotherapeuten nicht in den Sozialwissenschaften ausgebildet sind ${ }^{60}$, wie die Autoren meinen. Sie sehen daher auch nicht die Relevanz der sozialen Realität, die als solche Einfluss hat

60 Genau genommen müssten in diesem Zusammenhang auch die Geisteswissenschaften erwähnt werden. Philosophie, Literatur- und Geschichtswissenschaften erlauben andere, fruchtbare Zugangsweisen zum Verständnis der sozialen Realität. 
und unsere Vorstellungen von gelebter Individualität infrage stellen könnte. Möglicherweise löst auch die Gleichsetzung des Begriffes „sozial“ mit „sozialistisch", so die Autoren, eine solche Ablehnung aus (Hopper \& Weinberg, 2011, S. li f.).

\subsection{Repräsentationen des Unbewussten}

Wie man aus den bisherigen Ausführungen erkennen kann, ist das Unbewusste per definitionem nicht direkt zugänglich, es kann aber sehr wohl - wenn auch mit Einschränkungen - entschlüsselt werden. Auf der individuellen Ebene gehören dazu jene Äußerungsformen des Unbewussten, die im Traum, im Witz, in den Fehlleistungen, den Abwehrmechanismen, in Krankheitssymptomen oder in Übertragungs- und Gegenübertragungsprozessen zum Ausdruck kommen.

Mittels der Interviews wird zu klären sein, welche spezifischen Abwehrmechanismen in Auseinandersetzung mit der Finanz- und Wirtschaftskrise am Werk sind, welche Krankheitsbilder sie hervorbringen, wie mit Schuld und Schuldgefühlen umgegangen wird, wie man die auftretenden Ängste verstehen und zuordnen kann und ob Rückschlüsse auf das Kräfteverhältnis von Eros und Thanatos möglich sind. Sofern Träume und Witze präsentiert wurden, werden diese in die Analyse einfließen, genauso wie die in den Interviews wahrgenommenen Übertragungs- und Gegenübertragungsgefühle. Die von den Interviewpartnern verwendete Metaphorik, die sprachlichen Bilder und Modellvorstellungen, die mit der Krise und ihrem Ablauf in Verbindung gebracht werden, sollen im Hinblick auf ihre unbewussten Dimensionen einer Analyse zugeführt werden.

Auf einer kollektiven Ebene spielen Abwehrmechanismen ebenso eine Rolle; dennoch unterscheiden sich Gruppenprozesse, das Gruppenverhalten und Gruppendenken, wie sie etwa Tuckett (2013) am Beispiel der Trader von Finanzprodukten dargestellt hat ${ }^{61}$, von Vorgängen innerhalb eines Individuums oder Paares.

Mit Bezug auf Erdheim geht es in dieser Arbeit auch um das Aufspüren von Phantasmen im Krisendiskurs, also um Vorstellungskomplexe, die der Unbewusstmachung von Herrschaftsstrukturen dienen, oder, um mit Fromm zu sprechen, um die von Gruppen verdrängten Inhalte, die nicht ins Bewusstsein kommen dürfen, damit eine Gesellschaft reibungslos funktionieren kann (vgl. Fromm, 1962, S. 96).

61 Siehe dazu Kapitel 6.4. 
In der Analyse der Interviews wird der Schwerpunkt auf dem Individuum liegen; aber immer dann, wenn es um die Beschreibung von Gemeinsamkeiten oder Mustern geht, wird auf das gesellschaftlich/-e oder soziale Unbewusste als leitende Konzepte Bezug genommen werden.

Open Access Dieses Kapitel wird unter der Creative Commons Namensnennung 4.0 International Lizenz (http://creativecommons.org/licenses/by/4.0/deed.de) veröffentlicht, welche die Nutzung, Vervielfältigung, Bearbeitung, Verbreitung und Wiedergabe in jeglichem Medium und Format erlaubt, sofern Sie den/die ursprünglichen Autor(en) und die Quelle ordnungsgemäß nennen, einen Link zur Creative Commons Lizenz beifügen und angeben, ob Änderungen vorgenommen wurden.

Die in diesem Kapitel enthaltenen Bilder und sonstiges Drittmaterial unterliegen ebenfalls der genannten Creative Commons Lizenz, sofern sich aus der Abbildungslegende nichts anderes ergibt. Sofern das betreffende Material nicht unter der genannten Creative Commons Lizenz steht und die betreffende Handlung nicht nach gesetzlichen Vorschriften erlaubt ist, ist für die oben aufgeführten Weiterverwendungen des Materials die Einwilligung des jeweiligen Rechteinhabers einzuholen.

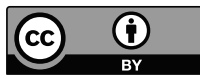

